\title{
Uncertainty quantification for deep learning in particle accelerator applications
}

\author{
Aashwin Ananda Mishra, Auralee Edelen, Adi Hanuka, and Christopher Mayes \\ SLAC National Accelerator Laboratory, Menlo Park, California 94025, USA
}

(Received 5 March 2021; accepted 15 November 2021; published 29 November 2021)

\begin{abstract}
With the advent of increased computational resources and improved algorithms, machine learning-based models are being increasingly applied to complex problems in particle accelerators. However, such datadriven models may provide overly confident predictions with unknown errors and uncertainties. For reliable deployment of machine learning models in high-regret and safety-critical systems such as particle accelerators, estimates of prediction uncertainty are needed along with accurate point predictions. In this investigation, we evaluate Bayesian neural networks (BNN) as an approach that can provide accurate predictions along with reliably quantified uncertainties for particle accelerator problems, and compare their performance with bootstrapped ensembles of neural networks. We select three accelerator setups for this evaluation: a storage ring, a photoinjector, and a linac. The problems span different data volumes and dimensionalities (e.g., scalar predictions as well as image outputs). It is found that BNN provide accurate predictions of the mean along with reliable estimates of predictive uncertainty across the test cases. In this vein, BNN may offer an attractive alternative to deterministic deep learning tools to generate accurate predictions with quantified uncertainties in particle accelerator applications.
\end{abstract}

DOI: 10.1103/PhysRevAccelBeams.24.114601

\section{INTRODUCTION AND MOTIVATION}

Particle accelerators represent some of the most complex and data intensive systems in the scientific realm. Herein, the interrelations between machine subsystems are complicated and often nonlinear, the system dynamics involve large parameter spaces that evolve over multiple relevant time scales that are often diverse, and accelerator systems can be difficult to model a priori. In this context, particle accelerators are well suited to data-driven approaches for modeling [1]. Some specific applications of machine learning in particle accelerators include anomaly detection [2], system modeling [3-5], virtual diagnostics [4,6,7], accelerator tuning and control [8-11], and others. While numerous machine learning algorithms are being explored by the accelerator community, the use of deep neural networks is becoming increasingly widespread. With their ability to act as universal approximators for complex relationships [12], process diverse data volumes and dimensionalities such as scalars, sequences, images as both inputs and outputs, and the availability of reliable

*aashwin@slac.stanford.edu

Published by the American Physical Society under the terms of the Creative Commons Attribution 4.0 International license. Further distribution of this work must maintain attribution to the author(s) and the published article's title, journal citation, and DOI. open-source tools, deep neural networks are seemingly opportune for accelerator applications.

Such deep learning models are often treated as highfidelity models with accurate predictive powers. However, even predictions from a trained neural network may contain significant errors and uncertainties due to bias, noise and complexity inherent in the data, the volume of the training data, the nature of the error minimum chosen during optimization, etc. The consequences of such errors and uncertainties are exacerbated by the lack of interpretability in deep learning models. Physics-based models often have clear physical interpretations for each term and thus, enable us to foresee and even correct some of these sources of error and uncertainty. However, deep learning models do not lend themselves to such straightforward interpretation. Our limited understanding of neural network mappings [13], coupled with the difficulty in verification and validation for complex deep neural network based models $[14,15]$, can lead to unforeseen consequences. For instance, deterministic neural networks are unable to recognize out-of-sample examples and habitually make erroneous predictions for such cases with high confidence [16-18]. Such uncertainty in predictions has had grave consequences while applying deep learning to high-regret applications. For example, the first fatality in automated driving systems occurred due to the inability of a trained AI-agent to differentiate the hue of a trailer from the color of the sky [19]. As a repeated theme, applying deterministic machine learning models in highregret and safety-critical systems can lead to undesirable 
outcomes [20-23], when accurate uncertainty estimates are not included to guide appropriate decision-making under uncertainty.

Data-driven modeling for particle accelerators poses additional challenges that compounds this potential for errors and uncertainties. These challenges arise due to the complex dynamics inherent to accelerators and the limitations of the data archives. With regard to the former, accelerators have high-dimensional parameter spaces wherein the dynamics are nonlinear and often even nonstationary. Additionally, anomalies in system responses can arise both shot-to-shot and over time, for instance due to drift, equipment failures, transients in equipment or incoming power sources. These features can introduce epistemic uncertainty in models as well as introducing dataset shifts while prediction. Beyond this, such data-driven modeling efforts have to deal with limitations of the data archives. Accelerator instrumentation is often limited due to cost constraints, thus resulting in missing data and important variables not being measured. The instrumentation, such as beam position monitors, and controllable components, such as rf sources, also have different levels of sensitivity and inherent noise for different beam parameters leading to heteroscedastic effects. Furthermore, this uncertainty is exacerbated by the presence of small, compounding errors in the descriptions of beamline elements, for instance, the magnetic fields of focusing magnets can have asymmetries that are difficult to quantify.

The need for robust modeling for particle accelerators is underscored by their applications. Particle accelerators are applied in fields where safety and reliability across disparate applications and diametric conditions are needed, such as medical therapy, scientific discovery, security, and defense. Numerous patients receive accelerator-based diagnoses and therapy each year for diseases like cancer [24]. Similarly, accelerators play an important role in national security, including cargo inspection, materials characterization, nuclear nonproliferation treaty verification, stockpile stewardship, etc., [25].

Thus, obtaining quantified uncertainties for machine learning models of accelerators is paramount if such models are to be applied in routine operation for prediction and control. For instance, high-power and high-intensity beams can lead to damage for essential and expensive components if not correctly controlled. Reliable uncertainties enable the operator to gauge the level of trust in surrogate model predictions, thus reducing such risk. Similarly, users of virtual diagnostics should have metrics available to them to indicate when the data-driven model has moved outside of its range of reliability and the predictions should not be relied upon. Similarly, in cases where models will be used to aid online control (for example, to provide an initial guess at optimal settings) or to aid offline experiment planning, it is essential that the uncertainty in the prediction be taken into account. SLAC is integrating machine learning-based models and modelbased control into operation of its accelerators. In this context, neural networks in particular are appealing for high dimensional sets of scalar or beam image inputs and outputs. However, we require reliable uncertainty estimates on neural network predictions across different accelerator setups, for their application in routine operations at SLAC.

Cases where neural network uncertainties are useful can be divided into three categories: instances of inexact prediction, out of distribution (OOD) samples, and adversarial examples. Instances of inexact prediction arise due to limitations of the data corpus and the network training. In accelerator applications, these are engendered due to the complex nonlinear relationships to be modeled in accelerators. With respect to OOD robustness, trained machine learning models are expected to maintain satisfactory performance when the inputs are hitherto unseen samples, that are drawn from the same distribution as the training data [26]. However, in real-life applications, this constraint is difficult to enforce and trained neural networks are compelled to make predictions for samples that may be significantly different from the training examples. In such cases, deterministic neural networks make predictions with a high degree of theoretical confidence. In accelerator applications, such instances can arise due to the nonstationary nature of the relationships to be modeled due to drift, regions of data sparsity due to the high dimension of the parameter spaces, and changes to the machine component configurations to support different experiments. Finally, robustness to adversarial examples will be important for the deployment of deep learning models in accelerator applications that are high risk targets (for example in the national security applications for particle accelerators [27-30]).

Some investigators have attempted to quantify uncertainties in deep learning models by utilizing model ensembles with randomization [31]. While model ensembling (with bootstrap aggregation and even random sub-sampling of features) improves predictive accuracy, there is limited mathematical basis to aver that this by itself would lead to reliable uncertainty estimates, encompassing both aleatoric and epistemic uncertainties. In literature, it has been shown that such straightforward model averaging may lead to under-estimation of uncertainty [32-35]. This under-estimation is further exacerbated in regions of the feature space with sparse data $[32,35]$. Such limitations can be alleviated by using ensembles of probabilistic models, as opposed to randomized ensembles of deterministic models [36].

In this context, Bayesian neural networks (BNNs) offer a probabilistic approach that scales to high-dimensional inputs and offer linear scaling with data volume. In contrast to conventional deterministic models, BNNs treat weights and biases as random variables with associated probability distributions. Bayesian learning of these weights is equivalent to changing the beliefs about the neural network (NN) 
weights from the prior distributions to the posterior distributions, as a consequence of observing the training data. As a direct consequence, this probabilistic approach provides a measure of uncertainty on the final model prediction. In essence, BNNs combine the accuracy and flexibility of deep learning approaches, with the uncertainty quantification inherent to the Bayesian formalism.

In this investigation, we apply BNNs to particle accelerator applications. We select problems across several accelerators at the SLAC National Accelerator Laboratory, in diverse data volume and data formats. We evaluate the ability of BNNs to provide accurate predictions of the mean, as well as generate reliable estimates for the predictive uncertainty. As a datum, we compare and contrast the performance of BNNs to bootstrapped ensembles of NNs. Further, we examine the performance of BNNs on the task of predicting uncertainties in beam phase space image predictions and their robustness to OOD samples.

The manuscript is divided into six sections. Following an overview of the problem in Sec. I, we introduce BNNs and bootstrapped ensembling in Sec. II. In Sec. III, we outline the performance of these two approaches across a variety of accelerator problems. In Sec. IV, we carry out tests to outline the robustness to OOD samples in probabilistic deep learning approaches. In Sec. V, we analyze the performance of BNNs for problems involving high-dimensional data input and output. The article concludes with a summary and recommendations for future steps in Sec. VI.

\section{MATHEMATICAL DETAILS}

In this section, we provide a brief outline of the two approaches to be compared (BNNs and ensembles) and the metrics used to compare them. In contrast to classical deterministic neural networks, in BNNs the weights and biases are assumed to be random variables with corresponding probability distributions. The training of BNNs involves inference of these probability distributions, conditioned upon the data. In this investigation, we utilize approximate Variation Inference to estimate the posterior distributions on these parameters. Our approach adheres to the Bayes By Backprop algorithm [37]. To optimize the variational parameters, we use the Adam algorithm, wherein the learning rate, the forgetting factors for gradients and second moments of gradients were set using cross validation.

In contrast to BNNs, bootstrapped ensembles utilize bootstrap aggregation for uncertainty estimation. Bootstrap datasets of $N$ samples each are produced for training each individual model in the ensemble by sampling uniformly with replacement from the original training dataset of $N$ unique samples. Individual neural networks in the model ensemble are trained separately on each of these bootstrap datasets. The predictions of the individual trained models in the ensemble are averaged to get a mean prediction. The variance in predictions provides a measure of the predictive uncertainty.

For the comparison, we aim for high accuracy of mean predictions, well-calibrated coverage from the prediction intervals, and minimal widths of prediction intervals (i.e., sharpness). To this end, we utilize three separate metrics. To assess the accuracy of the mean prediction, we utilize the mean absolute error (MAE), $\frac{1}{n} \sum_{i=1}^{n}\left|y_{i}-f\left(x_{i}\right)\right|$. The MAE for the bootstrapped ensembles and the BNN is contrasted to that of a singleton neural network in each case. Due to the ensembling inherent to each of these former approaches, it is expected that the MAE will be lower than the singleton deterministic neural network.

To ascertain the quality of the uncertainty estimates from the two approaches, the first metric used is the prediction interval coverage probability (PICP). In statistical literature, this is also referred to as coverage [38]. The prediction interval is the interval estimate provided by a model regarding the range in which the target value will lie with a certain probability, given the input features. If the actual value lies in the predicted interval, it is said to be covered by the interval leading to the concept of coverage by the prediction interval. The PICP measures the ratio of test samples that lie in the prediction interval. This also leads to the idea of calibration of prediction intervals. A critical consideration for prediction intervals is their reliability at reflecting the quantiles in the distribution of the target quantity. As an illustration, if a $95 \%$ prediction interval does not actually subsume $95 \%$ of the target samples, it can be misleading. Prediction intervals that exhibit lower PICP than expected indicated uncalibrated models. In the comparisons between bootstrapped ensembles and BNNs, we utilize the standard error as the uniform prediction interval.

To ensure sharpness of the prediction intervals, we utilize the mean prediction interval width (MPIW), which measures the average size of the prediction intervals over the test dataset. Sharpness is a valuable property because when the predictive distribution has correct calibration, a sharper distributional prediction is tighter around the observed data points and thus signifies more confidence in its predictions. It is expected that the prediction intervals provide a minimum required value of the PICP, while maintaining the MPIW to low values. To ensure equitable comparisons, the MPIW is measured at a fixed PICP. Here the prediction interval provided by the model is increased till a set PICP is reached and thence the MPIW is estimated. In the comparisons, we measure MPIW values at prediction intervals that ensure $90 \%$ coverage over the test dataset.

\section{COMPARISON OF DIFFERENT APPROACHES FOR ACCELERATOR TEST CASES}

In this section, we compare the performance of deterministic neural networks, bootstrapped ensembles of deterministic neural network models and BNNs on three specific particle accelerator problems, corresponding to 
(a)

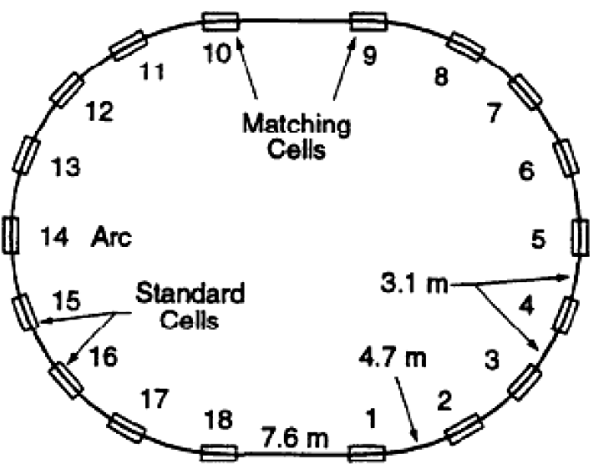

(b)

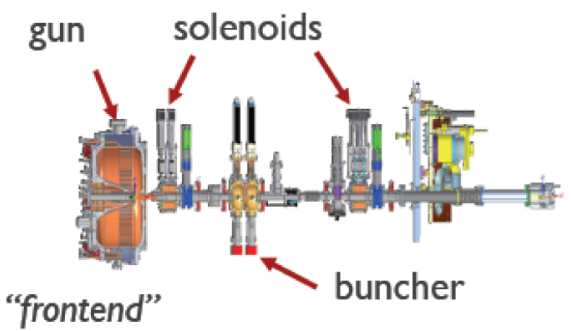

(c)

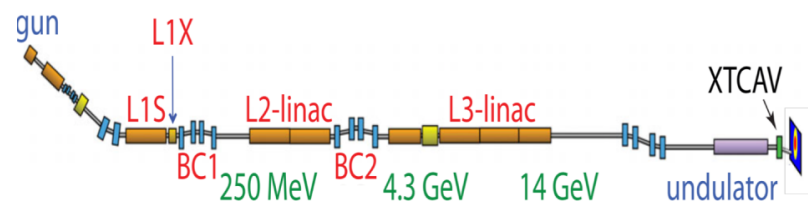

FIG. 1. Test cases for utilized in this investigation: (a) Schematic of the SPEAR3 storage ring, (b) the LCLS-II Injector frontend, and (c) the LCLS electron beamline.

cases outlined in Fig. 1. These are the SPEAR3 storage ring emittance prediction, prediction of beam parameters and distributions for the LCLS-II injector frontend, and prediction of beam parameters for the LCLS electron linac, at the entrance of the undulator. For each individual test case, identical training and testing datasets were used across all algorithms. The structure of the neural networks was also identical for all the algorithms. The other hyperparameters were separately optimized for each test case. While discussing the test cases, we commence with a discussion of the underlying accelerator problem, detail the data set in question, describe the hyperparameters for the models, and evaluate their performance.

\section{A. SPEAR3 ring}

SPEAR3 is a $3-\mathrm{GeV}$, high-brightness third generation storage ring operating with low emittance [39]. It runs with $500 \mathrm{~mA}$ in top-off mode, during which the beam current is maintained via the injection of electrons into the ring every 5 min.

The emittance is a measure of the phase space area occupied by a particle beam in one degree of freedom of beam motion. Smaller emittances are preferred in storage ring light sources as they result in high brightness of the photon beam. Ideally, the vertical emittance of an electron storage ring is nearly zero. However, there are many factors that lead to finite vertical emittances in an operating electron storage ring. These include the vertical dispersion generated by magnetic and installation errors, and betatron coupling between the horizontal and vertical planes [40]. These sources of error contributing to vertical emittance may be compensated with skew quadrupole magnets. In SPEAR3, there are 13 free skew quadrupoles for vertical emittance control. These are schematically outlined in Fig. 1(a) [41]. The free skew quadrupoles are located in Secs. $1,2,3,5,7,8,9,10,11,12,14,17$, and 18 . Usually the skew quadrupole setting for vertical emittance correction is obtained by fitting the orbit response matrix data [42], in particular, the off-diagonal elements. Since beam loss is dominated by Touschek scattering for most third generation light sources, the beam loss rate provides an indirect way to minimizing vertical emittance. In such cases, the square root of the vertical emittance is inversely proportional to the beam loss rate. Consequently, minimizing vertical emittance is equivalent to maximizing beam loss rate with respect to the skew quadrupoles. This methodology for minimization of vertical emittance with skew quadrupoles using loss rate as the indirect measure has been illustrated in prior research [43].

The first dataset for this case consisted of 650 direct experimental measurements of the beam loss rate (in $\mathrm{mA} /$ min) along with the corresponding parameters for the 13 free skew quadrupoles. The beam loss rate is measured by monitoring the beam current drop in a fixed time interval. The skew quadrupole parameter current range is $(-20,20)$ A. Along with the intrinsic epistemic uncertainty, there is aleatoric uncertainty inherent to the problem as noise in the dataset arising due to the uncertainty in the beam current measurement. The original learning dataset of 650 samples was divided into a training set, of 520 samples, and a testing set of the remaining 130 samples. The target variable is the beam loss rate, and, the features acting as inputs to the model are the parameters for the 13 free skew quadrupoles.

The deterministic NN consisted of seven hidden layers with eight neurons each. The activations across all neurons were the rectified linear units (ReLU). The network architecture and hyperparameters were tuned manually using cross validation. In the bootstrapped ensemble of networks, the number of NNs in the ensemble were increased till the PICP and MPIW became constant beyond a value of 25 individual NNs in the ensemble. For the BNN, the architecture was selected as having seven hidden layers with eight neurons each. The input layers has 13 features and the output is a scalar. Following best practice, all weights and biases in the network were initialized with a standard normal prior.

The predictions of the different models are outlined in Fig. 2. In the figure, the test data samples are sorted in ascending order of the beam loss rate while plotting the 

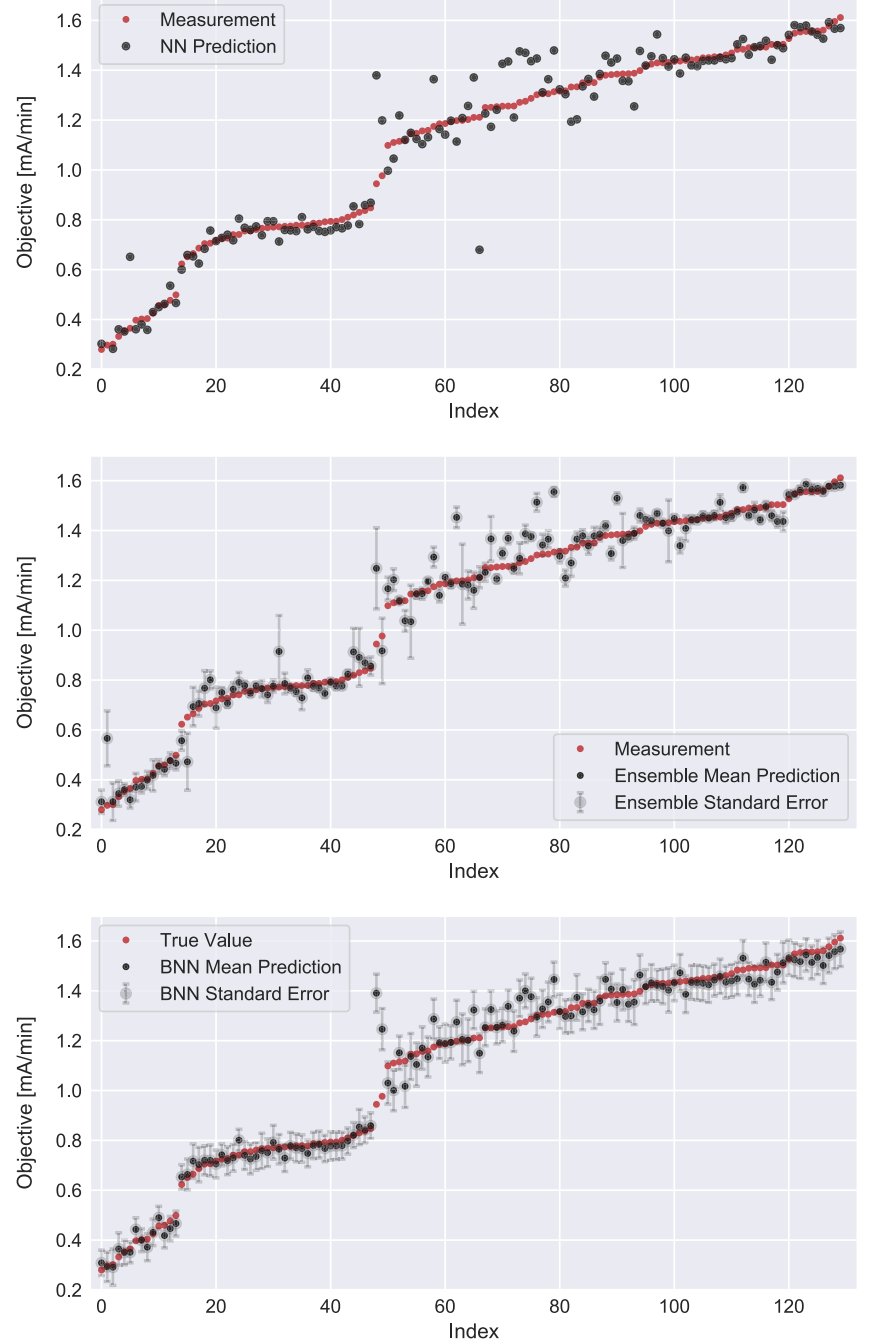

FIG. 2. Deterministic neural network, ensemble and Bayesian neural network model mean predictions and predicted standard error for SPEAR3 measured data.

results to aid in visualization. While the deterministic NN predictions are reasonably accurate, there are samples with intermediate values of the beam loss rate where the predictions have significant discrepancy. The mean prediction of the bootstrapped ensemble shows improvement in accuracy compared to the individual deterministic $\mathrm{NN}$, as outlined in Table I. However, the prediction uncertainties indicated by the ensemble do not reflect the discrepancy in predictions. There are many samples that have high discrepancy in predictions, but the standard error outputted by the bootstrapped ensemble is negligibly small. The BNN mean predictions also exhibit higher accuracy as compared to the deterministic $\mathrm{NN}$, due to the ensembling effect. However, the uncertainty intervals indicated by the BNN are more reflective of model discrepancy. The standard error prediction interval for the BNN exhibits a coverage of 0.82 , as compared to 0.49 for the bootstrapped ensemble. Additionally, at a PICP of 0.90, the MPIW for the BNN is
TABLE I. Comparison of models for SPEAR3 measured data.

\begin{tabular}{llcc}
\hline \hline Model & MAE & PICP & MPIW \\
\hline DNN & 0.06 & $\ldots$ & $\ldots$ \\
Ensemble & 0.043 & 0.49 & 0.24 \\
BNN & 0.034 & 0.82 & 0.11 \\
\hline \hline
\end{tabular}

substantially lower than the bootstrapped ensemble, as outlined in Table I. Thus, the uncertainty intervals from the BNN provide better coverage and are sharper.

To test the ramifications of data volume on the relative performance, the analysis was carried out over a larger dataset. This dataset corresponds to a simulation of the SPEAR3 ring and consists of 3500 samples of the beam loss rate, and, the corresponding parameters for the 13 free skew quadrupoles. The training dataset from the simulation data was limited at 3000 samples, along with 250 samples each for the test and validation datasets.
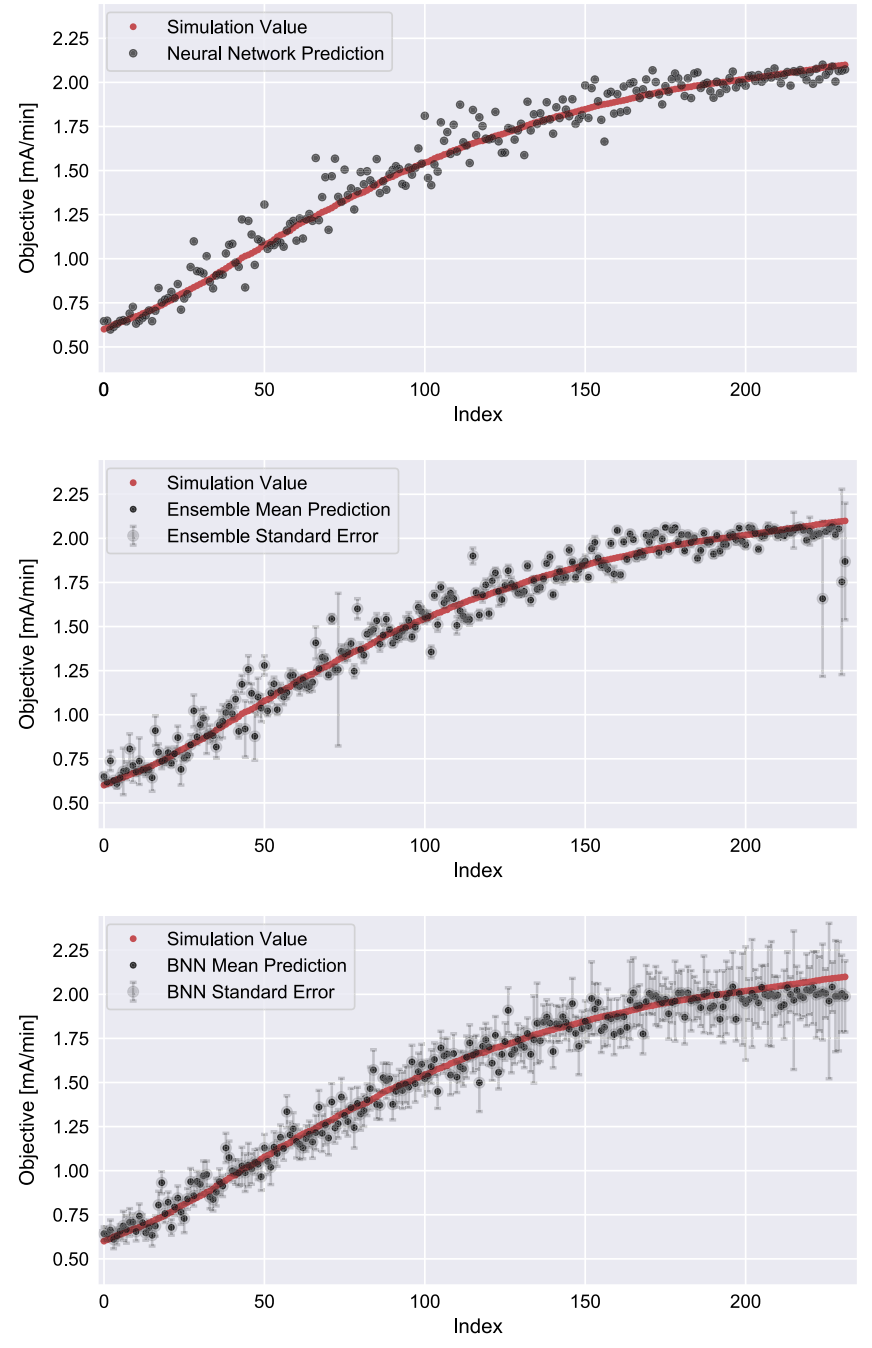

FIG. 3. Deterministic neural network, ensemble and Bayesian neural network model mean predictions and predicted standard error for SPEAR3 simulation data. 
TABLE II. Comparison of models for SPEAR3 simulation data.

\begin{tabular}{llcc}
\hline \hline Model & MAE & PICP & MPIW \\
\hline DNN & 0.07 & $\ldots$ & $\ldots$ \\
Ensemble & 0.065 & 0.45 & 0.36 \\
BNN & 0.055 & 0.81 & 0.19 \\
\hline \hline
\end{tabular}

Representative predictions for the models on the test dataset for the simulation data are outlined in Fig. 3. It is observed that the deterministic NN predictions are reasonably accurate, but include test samples where there is significant difference between the NN prediction and the simulation results. The mean prediction from the bootstrapped ensemble exhibits marginal increase in accuracy, as outlined in Table II. However, the prediction intervals outputted by the ensemble have low coverage, as there are test samples where the mean prediction is accurate but have a large variance in prediction and vice versa. The coverage of the standard error interval for the bootstrapped ensemble is 0.45 over the test dataset. The uncertainty estimates predicted by the BNN are more indicative of model discrepancy, as outlined in Fig. 3. At low values of the beam loss rate objective, the predictions are largely accurate and the predicted standard error is low. At high values of the beam loss rate, the predictions of the BNN model exhibit lower accuracy. However, for this range the predicted standard error is relatively larger as well. The standard error interval from the BNN provides 0.81 coverage and thus, the prediction intervals are well calibrated.

In addition to the coverage and the sharpness of the resultant prediction intervals, a pragmatic concern is the computational cost associated with the algorithm to engender uncertainty estimates. To this end, we compare the empirical efficiency and the computational cost of employing bootstrapped ensembles of NNs against variation inference-based BNNs by comparing the CPU times of the runs. For the SPEAR3 measured dataset, the CPU times (in seconds) for the BNN is $4190 \mathrm{~s}$ and for the Bootstrapped ensemble is 5925 s. For the larger SPEAR3 simulation dataset, the CPU time for the BNN is $8646 \mathrm{~s}$, while the Bootstrapped ensemble required $12842 \mathrm{~s}$.

Thus, it can be observed that the training of the $\mathrm{BNN}$ is computationally more expensive than a singleton deterministic NN by over an order of magnitude. However, while using Bootstrapped ensembles of deterministic NNs, the aggregation of training times over the individual models in the ensemble increases the computational expense. In this light, the computational expense of training the BNN using variation inference is comparable to training an ensemble of bootstrapped deterministic NNs. In should be pointed out that the training of individual models in the bootstrapped ensemble is easily parallelizable. Doing so would reduce the wall time for training the ensemble, but have significantly lesser impact on the CPU time.
TABLE III. Details of wide scans over features for the LCLS-II injector dataset.

\begin{tabular}{lcccc}
\hline \hline Variable & Min value & Max value & Nominal & Unit \\
\hline Gun phase & -10 & 10 & -6.6 & $\mathrm{deg}$ \\
Solenoid 1 & 0 & 0.1 & 0.06 & $\mathrm{~T}$ \\
Solenoid 2 & 0 & 0.1 & 0.03 & $\mathrm{~T}$ \\
Buncher amplitude & 0 & 2 & 1.80 & $\mathrm{MV} / \mathrm{m}$ \\
Buncher phase & -100 & -60 & -80.3 & $\mathrm{deg}$ \\
\hline \hline
\end{tabular}

\section{B. LCLS-II injector}

In this sub-section, we focus on the modeling of the LCLS-II injector. The modeling of injectors is often more difficult due to the nonlinear effects at low energy (e.g., space charge). The simulation dataset consists of scans of the gun phase, the strengths of the two solenoids, and the buncher amplitude and phase using the simulation code IMPACT [44]. The ranges for each of the input features in the dataset over this wide scan, along with their minimum, maximum, and nominal values are reported in Table III. The desired outputs ranged over two scalar outputs and longitudinal phase space (LPS). For the purposes of this discussion, we focus on the scalar $x$-emittance target, $\varepsilon_{x}$. The learning dataset consisted of 4000 samples for training, 200 for validation, and 110 for testing.

For the NNs, the architecture was selected as having eight densely connected hidden layers, each with ten neurons. The input layer has five features and the output is a scalar. The activations across all neurons were the ReLU. The network architecture and hyperparameters were tuned manually using cross validation. In the bootstrapped ensemble of networks, the number of NNs in the ensemble were increased till the PICP and MPIW became constant beyond a value of 30 individual NNs in the ensemble. Following best practice, all weights and biases in the BNN were initialized with a standard normal prior.

Outputs from the different models are outlined in Fig. 4. The deterministic NN predictions are accurate across the range of emittance but inaccurate near the upper and lower limits of this target. This can be explained by observing the distribution over the emittance in the learning dataset, reported in Fig. 5. As can be observed in Fig. 5, there is a paucity of samples near the upper and lower limits of the $x$-emittance target. This is a feature common to generated datasets, where we can engender the sampling in feature space to be uniform and fine grained. However, this does not lead to a uniform sample over the target and can lead to models that are inaccurate in many regions of the target. It is observed that the mean predictions of the bootstrapped ensemble do not show significant improvement over the singleton deterministic NN, also reported in Table IV. Additionally, the uncertainty estimates from the bootstrapped ensemble do not reflect the discrepancy in the predictions of the ensemble. For instance, many of the test 

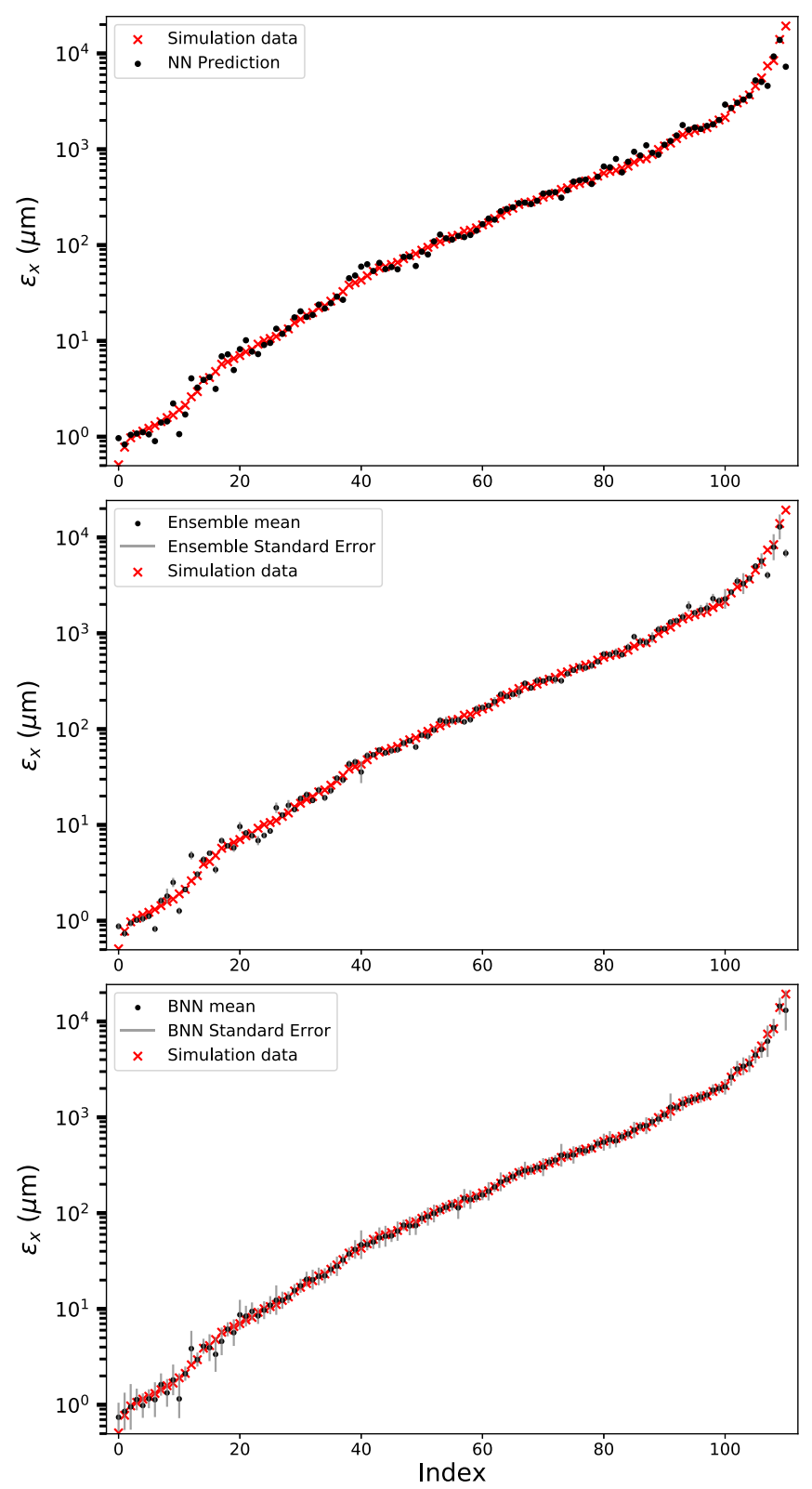

FIG. 4. Deterministic neural network predictions; Ensemble and Bayesian neural network model mean predictions and predicted standard error for LCLS-II injector front end simulation data.

samples near the upper and lower limits of this target are inaccurately predicted by this ensemble model but the predictive uncertainty for those samples is small. This may be a result of the bootstrapping approach where every individual NN in the ensemble has access to only approximately a proportion of 0.63 of the original training dataset. In this scenario, very few of the training samples from regions with low target density are accessed by the individual models in the ensemble and the ensemble model is ill calibrated in these regions. The training of the BNN model has access to all the samples in the training dataset, and it shows improved performance with respect to the accuracy of the mean prediction. Additionally, the standard

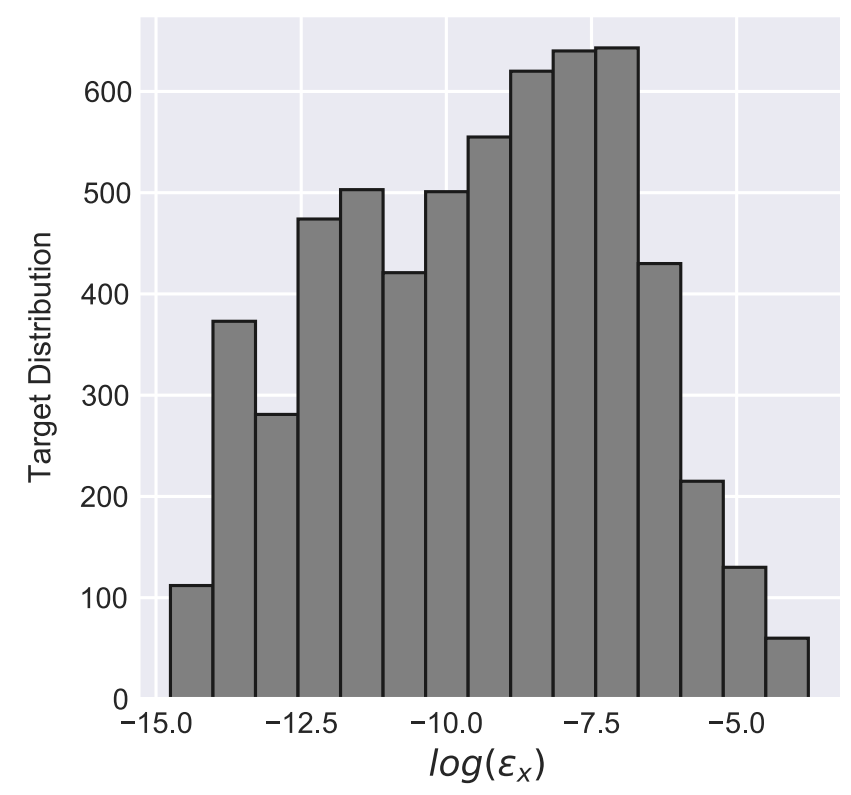

FIG. 5. Histogram outlining the distribution of log emittance in the learning dataset.

error bound predicted by the BNN shows a coverage of 0.77 over the test dataset, as compared to 0.39 for the bootstrapped ensemble as shown in Table IV. In this context, the prediction intervals outputted by the BNN are better calibrated and more reliable.

Addressing the computational cost of utilizing bootstrapped ensembles and BNNs, we find that the CPU times (in seconds) for the BNN is $9930 \mathrm{~s}$ and for the bootstrapped ensemble is $12030 \mathrm{~s}$. Thus, both the approaches have comparable computational expenses.

\section{LCLS electron beamline}

The LCLS electron beamline is outlined in Fig. 1(c). Herein, the Linac 1 and L1S phase set points influence both the electron bunch energy and the length change engendered by the bunch compressor, BC1. A substantial change of L1S due to drift has to be corrected via a phase scan. The Linac $1 \mathrm{X}$-band, L1X, cavity phase set point linearizes the LPS, compensating for energy curvature produced by the Linac 1. The bunch compressor 1, BC1, energy set point determines the amount of longitudinal compression of the bunch and provides feedback for the L1 amplitude set point. The Linac 2, L2, phase set point controls a set of klystrons and effects bunch length. This suffers from the same drifts as L1. The bunch compressor 2, BC2, energy

TABLE IV. Comparison of models for LCLS-II injector.

\begin{tabular}{lccc}
\hline \hline Model & MAE & PICP & MPIW \\
\hline DNN & 0.119 & $\ldots$ & $\ldots$ \\
Ensemble & 0.103 & 0.39 & 0.562 \\
BNN & 0.098 & 0.77 & 0.244 \\
\hline \hline
\end{tabular}


TABLE V. Details of wide scans over features for the LCLS beamline dataset.

\begin{tabular}{lcccc}
\hline \hline Variable & Min value & Max value & Nominal & Unit \\
\hline L1 phase & -40 & -20 & -25.1 & deg \\
L2 phase & -50 & 0 & -41.4 & deg \\
L3 phase & -10 & 10 & 0 & deg \\
L1 voltage & 50 & 110 & 100 & percent \\
L2 voltage & 50 & 110 & 100 & percent \\
L3 voltage & 50 & 110 & 100 & percent \\
\hline \hline
\end{tabular}

set point is a second stage of compression at higher energy. The Linac 3, L3, phase set point is another klystron system and is similarly subject to phase and amplitude drifts. The learning dataset for the LCLS electron beamline was simulated using Bmad [45], and included all the prominent nonlinear beam effects up to the undulator entrance. The dynamics of electron bunches in such free electron lasers are affected by complex effects such as time varying beam phase space distributions, misalignments, and collective effects such as coherent syncrotron radiation, cavity structure wakefields.

The learning dataset for the LCLS electron beamline is a random sample over a wide range of the linac phases and voltages (six variables, consisting of the voltages and phases of L1, L2, and L3), that are adjusted to manipulate the beam's shape in LPS. The ranges for each of the input features in the dataset over this wide scan, along with their minimum, maximum and nominal values are reported in Table V. The sampled data covers a majority of the operating range of the LCLS. The training data consisted of 3500 samples, the validation set of 300 samples and the testing data, 200 samples.

For the NNs, the architecture was selected as having eight hidden layers that are densely connected, each with eight neurons. The input layers has six features and the output is a scalar, specifically predicting the natural logarithm of the emittance along the $x$ axis, $\varepsilon_{x}$. The activations across all neurons were the ReLU. The network architecture and hyperparameters were tuned manually using cross validation.

In Fig. 6(a), the predictions of the deterministic NN for this target are reported. At low values of the emittance, the model's predictions are relatively accurate, but deteriorate at high values of the emittance. The rationale underlying this can be inferred from the distribution of the learning dataset, outlined in Fig. 7. We observe that while many samples are present in the dataset at low emittance values, these become more scarce at higher values. Thus, random sampling over the entire feature space does not lead to a uniformly sampled learning dataset over the range of the target.

The mean predictions of the bootstrapped ensemble show significant improvement in accuracy over the deterministic NN, as is also reported in Table VI. However, the

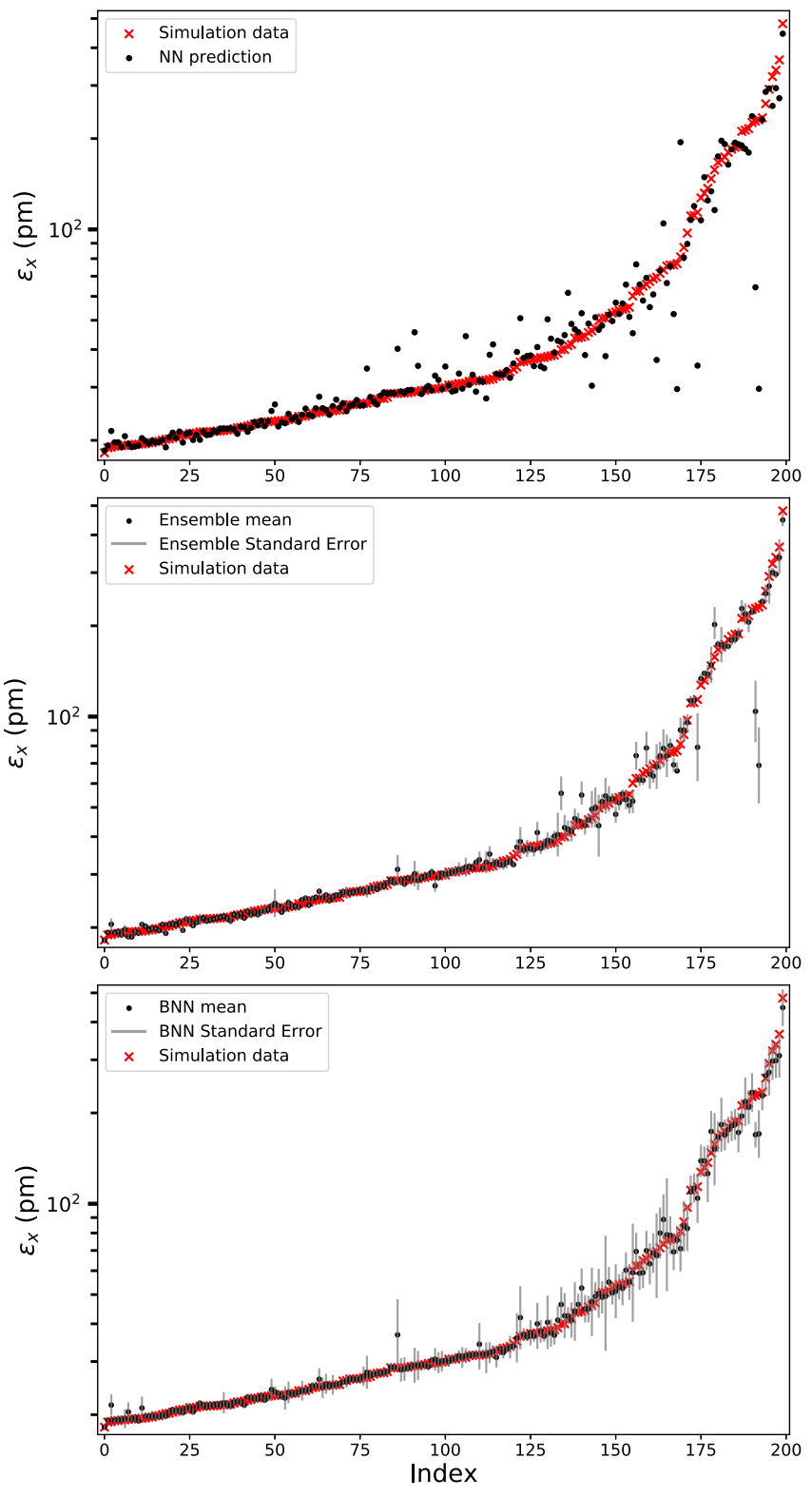

FIG. 6. Comparison of (a) Deterministic neural network, (b) Bootstrapped ensembles, (c) Bayesian neural network predictions for the $x$-emittance for the LCLS electron beamline.

uncertainty estimates from the bootstrapped ensemble are often not indicative of the discrepancy in predictions. The coverage from the standard error interval of the bootstrapped ensemble is only 0.48 , which indicates that the prediction intervals are ill-calibrated. The accuracy of the BNN is comparable to the bootstrapped ensemble. However, the coverage from the BNN prediction intervals is 0.75 over the test dataset, which indicated that the BNN prediction intervals are well calibrated. Additionally, the MPIW for the BNN is significantly lower than the bootstrapped ensemble (Table VI), indicating sharper prediction intervals.

With respect to the computational costs of utilizing bootstrapped ensembles and BNNs, the CPU times (in 


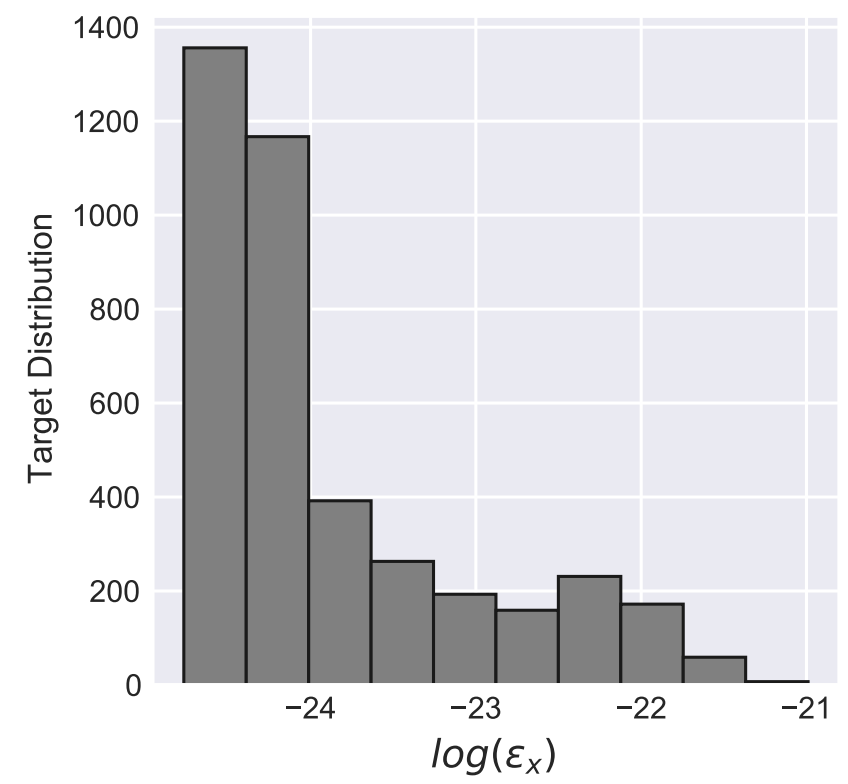

FIG. 7. Histogram outlining the distribution of log emittance in the learning dataset.

seconds) for the BNN is $17450 \mathrm{~s}$ and for the Bootstrapped ensemble is $19320 \mathrm{~s}$. Thus, both the approaches have comparable computational expense.

\section{OOD ROBUSTNESS}

An inherent assumption in machine learning is that the samples to be predicted should adhere to the same probability distribution as the training dataset. Samples where this condition is met are referred to as in-sample instances, $p_{\text {samples }}(X, Y)=p_{\text {training }}(X, Y)$. Samples where this condition is violated are referred to as OOD instances, $p_{\text {samples }}(X, Y) \neq p_{\text {training }}(X, Y)$. Such a change in the joint distributions of the training data compared to that of the prediction samples is termed dataset shift [46]. This can be manifested in different forms, such as covariate shift (when the distribution over feature space, $p(X)$ changes but the conditional distribution is unchanged), label shift (when the distribution over target space, $p(Y)$ changes but the conditional distribution is unchanged), open set recognition (when new classes or ranges are present in the target space of the prediction sample), etc. In particle accelerator applications, such dataset shifts are manifested often. For instance, the trained surrogate models can be used for exploration of the parameter space that is beyond the range of the training data. Additionally, there can be a change in

TABLE VI. Comparison of models for LCLS beamline.

\begin{tabular}{lccc}
\hline \hline Model & MAE & PICP & MPIW \\
\hline DNN & 0.049 & $\ldots$ & $\ldots$ \\
Ensemble & 0.028 & 0.48 & 0.25 \\
BNN & 0.025 & 0.75 & 0.16 \\
\hline \hline
\end{tabular}

the nature of the data, for instance using a surrogate model that is trained on simulation data and directly deployed to make predictions in real life applications. Herein, there is a difference between the inferred behavior of the model from simulation data and the actual machine system, as simulations rely on simplifications and often represent idealized beam dynamics. Another key concern is the temporal drift in the machine system's response to given inputs. This can be manifested due to equipment aging, replacement of parts, environmental changes, etc. In such cases, the conditional distribution of the target changes due to drift. In all the aforementioned cases, deterministic surrogate models provide overly confident predictions that may be misleading. Traditional deep NNs are particularly susceptible to such dataset shifts and make overconfident and even erroneous predictions on samples that are substantially different from the training data $[17,34,47]$. In such a scenario, predictive uncertainty estimates are critical to ascertain when the model predictions can be reliable and robust to dataset shifts.

While different covariate shifts and perturbations can be introduced in image inputs, we engender an instance using the SPEAR3 simulation data discussed in Sec. III A. The thirteen dimensional input features are projected onto a two-dimensional space defined by their principal axes, using PCA. In this lower dimensional representation, $\mathrm{k}$-means clustering is utilized to split the data into three clusters, outlined in Fig. 8. The two clusters with maximal distance between cluster centers are selected as the learning dataset and the OOD dataset, as outlined in the figure, while the cluster abutting both these is removed. There is significant separation in the cluster centers of the learning dataset and the OOD dataset, and they have no overlap in this lower dimensional space. The learning dataset is split into a training dataset with $80 \%$ of the samples and $20 \%$ in an in-sample test dataset. We train deterministic NNs and BNNs on the training dataset. Their performance is evaluated on the test dataset that is in-sample and the OOD dataset. The network architecture and other hyperparameters are identical to those used in Sec. III A.

The performance of the deterministic $\mathrm{NN}$ is outlined in Fig. 9. The NN predictions are reasonably accurate on the

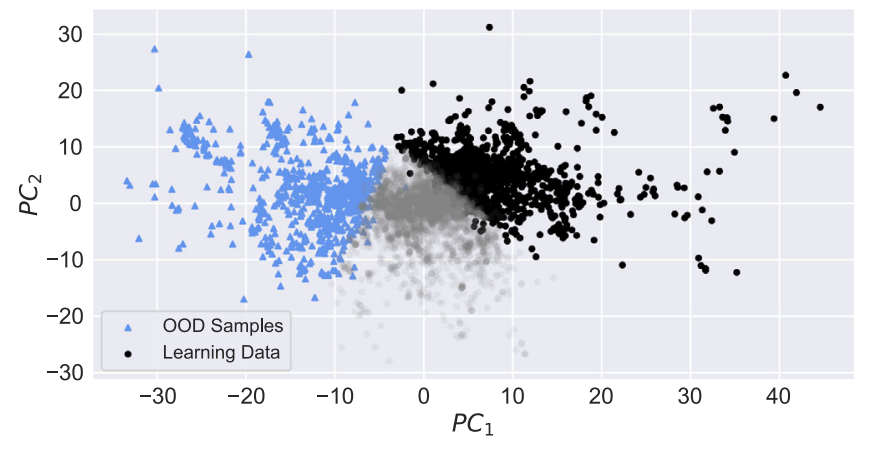

FIG. 8. Outlining the clusters of data using PCA. 

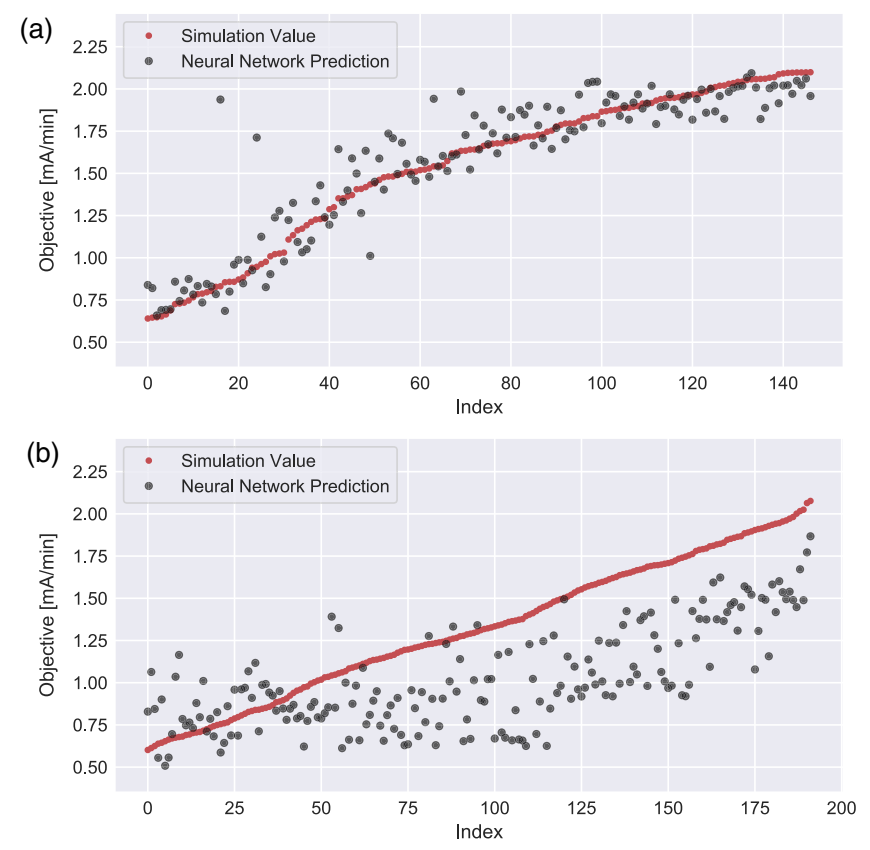

FIG. 9. Comparison of deterministic NN predictions on (a) insample test dataset samples and (b) OOD dataset samples.

in-sample test dataset samples. However, the NN predictions are significantly inaccurate on the OOD dataset samples. The results for the BNN are reported in Fig. 10. The performance of the mean predictions of the BNN are substantially depreciated when the predictions are made on the OOD samples, as compared to the in-distribution test
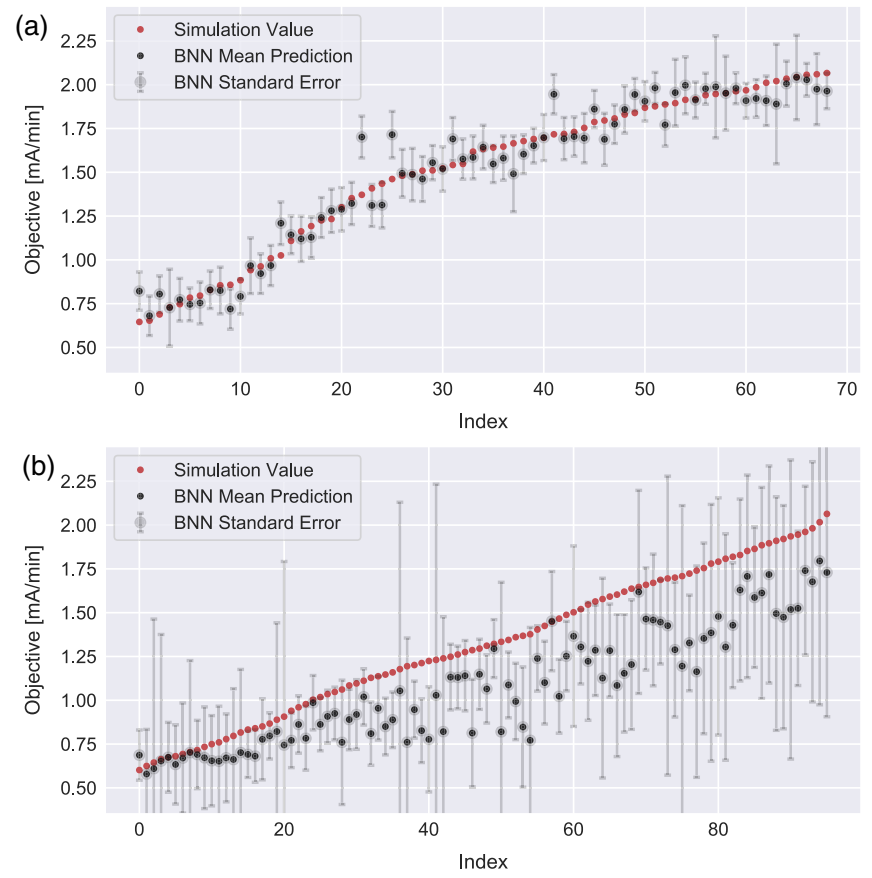

FIG. 10. Comparison of Bayesian neural network predictions on (a) in-sample test dataset samples and (b) OOD dataset samples. dataset. However, the uncertainty outputted by the BNN increases significantly on the OOD dataset contra the insample test dataset. The MPIW on the in-sample test dataset is 0.16 and on the OOD dataset is 0.35 . Furthermore, as the OOD dataset points move away from the learning dataset cluster center, the predictive uncertainty trends higher. In such a scenario, BNNs show OOD robustness, where cutoffs can be set on their prediction intervals to denote samples where the surrogate model may be relied upon and samples for which the surrogate model predictions may be unreliable.

\section{EXTENSION TO PREDICTION OF BEAM PHASE SPACE IMAGES}

In addition to scalar quantities, prediction of beam phase space projections are often used to provide additional information about the beam. The beam itself is a collection of six-dimensional information (three positions and three momenta for each particle). Two-dimensional projections of the phase space can be directly measured. Thus, for NN models of accelerator systems, predictions of both the phase space projections and their uncertainty is useful. In addition to being useful for general accelerator modeling (e.g., for offline optimization and experiment planning), ML-based image predictions are useful for providing noninvasive estimates of the beam phase space in cases where it cannot be continuously measured [7]. Quantifying the confidence in the prediction and specifically, having reliable estimates of uncertainty across different regions of the LPS image predictions can be a critical guide to decision-making. Herein, we focus on prediction of the LPS images of the LCLS-II injector. To generate the dataset, simulations on ASTRA [48] were carried out

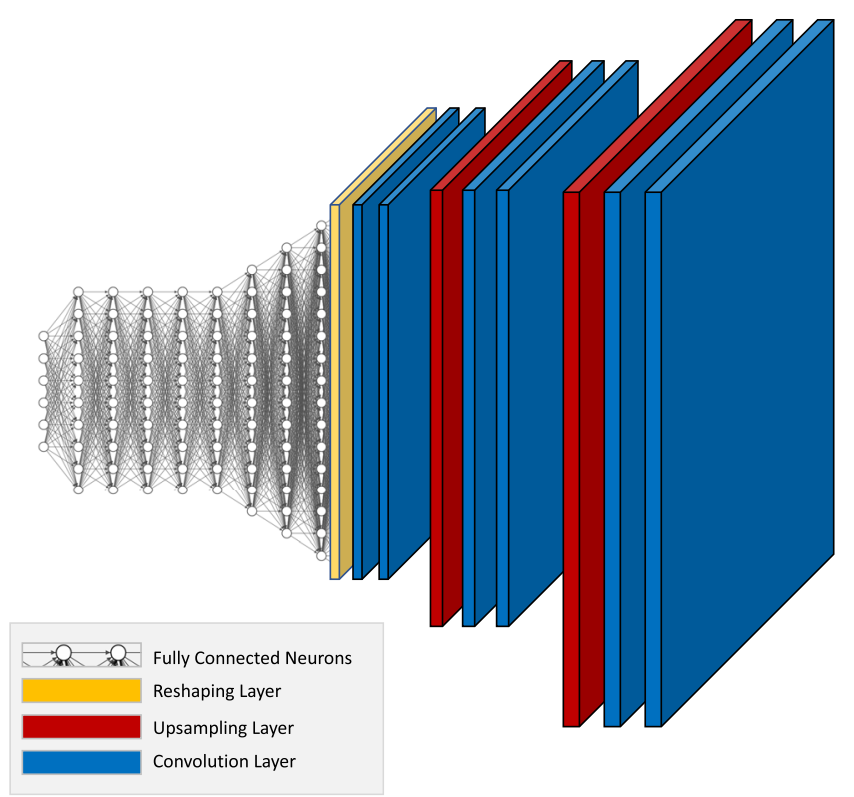

FIG. 11. Schematic for the encoder-decoder architecture for the LCLS-II injector longitudinal phase space image prediction. 

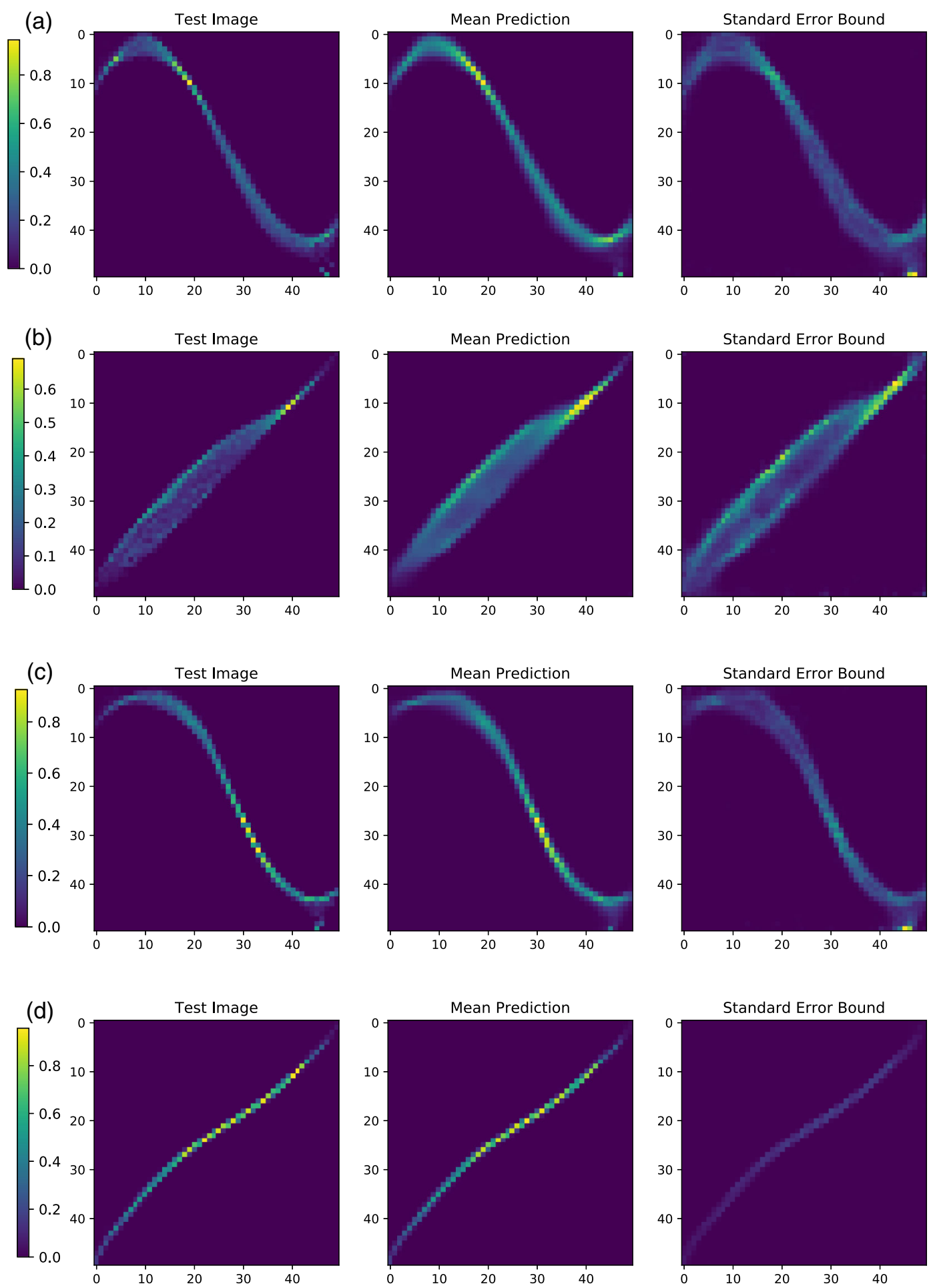

FIG. 12. Simulation test images, Bayesian neural network predictions and predicted standard error bounds for the LCLS-II injector longitudinal phase space.

and the input features consisted of scalar inputs generated by scanning the gun phase, two solenoid strengths, and the buncher amplitude and phase. The network architecture consisted of encoder and decoder sections. The encoder section consists of nine densely connected layers with the five scalars serving as inputs. The successive fully connected layers in the encoder section had 20, 20, 20, $20,20,100,200,600$, and 10000 neurons, respectively. The vector output of the encoder section is reshaped into a higher-order tensor before being fed into the decoder section. The decoder section consists of two sets of two convolutional layers (consisting of $164 \times 4$ filters each), 

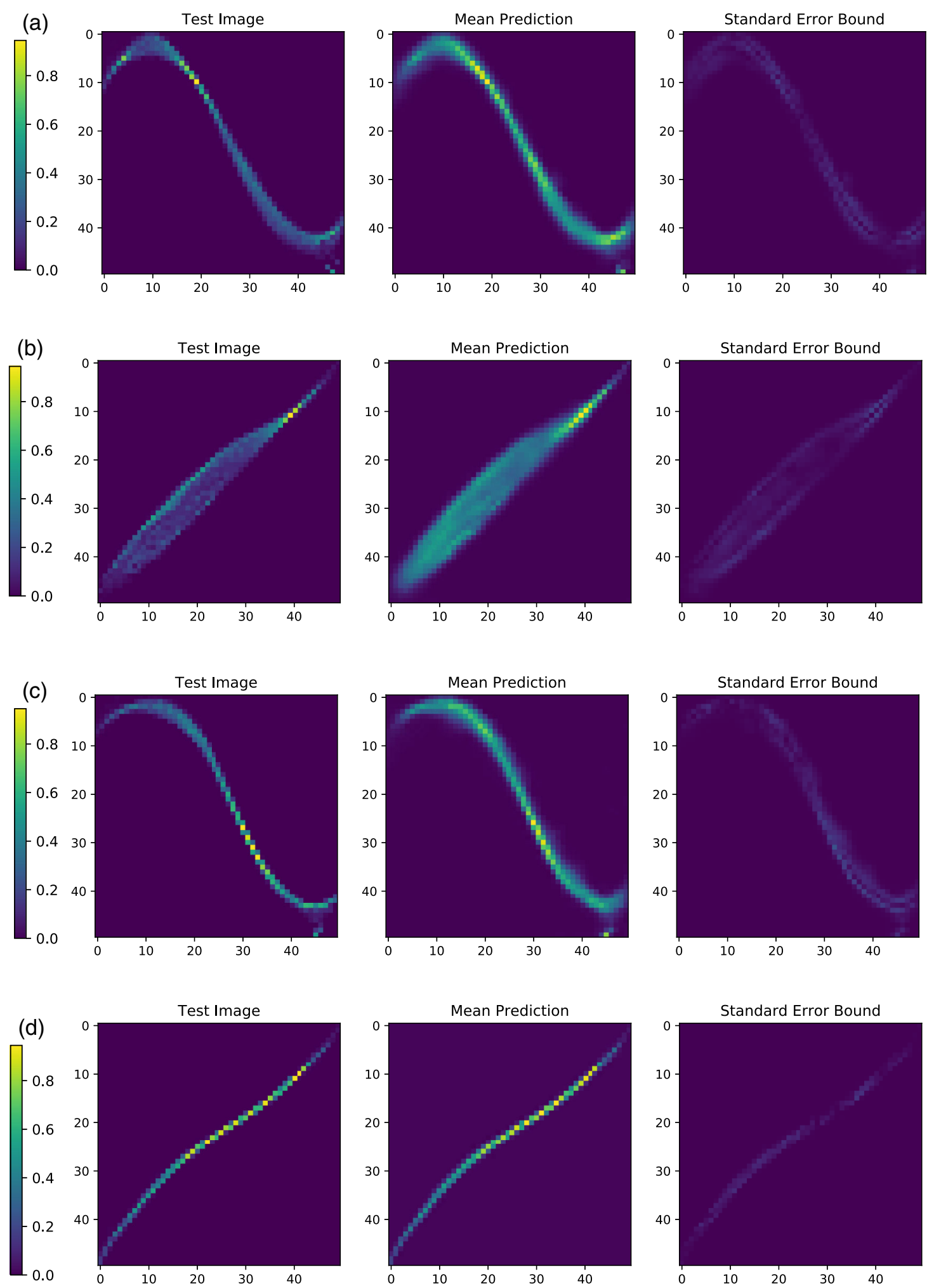

FIG. 13. Simulation test images, neural network ensemble predictions and predicted standard error bounds over the ensemble of deterministic neural networks for the LCLS-II injector longitudinal phase space. (a), (b), (c) and (d) are representative instances, randomly chosen.

followed by an upsampling layer $(2 \times 2$, the upsampling factor for both rows and columns in the input is 2). This is followed by two final convolutional layers engendering the output image. The network architecture is schematically outlined in Fig. 11. The training dataset consisted of 15,000 pairs of scalar inputs and image outputs. The testing dataset 
consisted of 4000 pairs of scalar inputs and image outputs. The model architecture was manually tuned using cross validation.

Outputs from the trained BNN are outlined in Fig. 12 for a set of representative examples from the test dataset. For each instance, the test image from the simulation, the mean prediction from the $\mathrm{BNN}$, and the standard error predicted by the BNN are reported. The standard error highlights regions where there is significant discrepancy between the mean prediction and the simulation output, for instance in Fig. 12(c). In cases where the mean prediction is in close agreement with the simulation, for instance in Fig. 12(d), the standard error is correspondingly lower. The corresponding outputs obtained from an ensemble of deterministic NNs are reported in Fig. 13. An ensemble of 15 deterministic NNs was trained on bootstrapped subsets of the training dataset. The architecture of the networks was the same as has been described for the Bayesian network. Beyond 15 bootstrapped models in the ensemble, the ensemble mean prediction and variances do not show significant changes with additional models. The mean predictions from the bootstrapped ensemble are similar in accuracy to the BNN. However, there is a marked difference in the reliability of the uncertainty bounds. The variance of the bootstrapped ensemble predicts small standard errors over the image output, irrespective of the discrepancy between the mean prediction and the simulation output. This is in contrast to the BNN, wherein the predicted standard error over the image output was indicative of the magnitude and the distribution of this discrepancy. With respect to quantitative metrics, the MAE for singleton NNs was 0.008 , for the bootstrapped ensemble it was 0.002 and for the BNN the MAE was 0.003. However, the coverage from the standard error interval of the bootstrapped ensemble is 0.78 , while for the BNN standard error predictions the coverage is 0.94 . This suggests that the uncertainties from the BNN may be more reliable.

\section{SUMMARY \& CONCLUSIONS}

With the advent of increased computational resources, improved algorithms, and the availability of software tools, machine learning based models are being increasingly applied to complex problems in particle accelerators. In this context, considering the complicated nature of the relationships to be modeled, the large corpora of data and the high dimensionality of the inputs and outputs, deep learning tools are gaining popularity in accelerator applications.

While deep learning models have obvious modeling capabilities, they are prone to overfitting and may provide unduly confident predictions with unknown errors and uncertainties. For reliable deployment of deep learning algorithms in high-regret and safety-critical systems such as particle accelerators, quantification of the predictive uncertainty is required. To this end, investigators have utilized bootstrapped ensembles of NNs to quantify predictive uncertainty. However, some investigations have shown that using bootstrap aggregation with deterministic NNs may lead to unreliable predictions of uncertainty $[32,49]$. Additionally, in regions with significant data sparsity, bootstrapping leads to limited benefits [50,51].

In this investigation, we evaluated BNNs as an alternative approach that can provide accurate predictions of the mean behavior, along with reliable uncertainty quantification for particle accelerator problems. We select problems across different designs, including both a ring-based and a linac-based accelerator, in diverse data volume and data formats, e.g., scalar predictions as well as images. It was shown that BNNs provide reliable predictions of the mean along with reliable estimates of predictive uncertainty across the test cases. Additionally, BNNs provide robustness to OOD samples. In this vein, BNNs may offer an attractive recourse to deterministic deep learning approaches, to generate accurate predictions with quantified uncertainties across particle accelerator applications.

\section{APPENDIX A: BACKGROUND ON UNCERTAINTY QUANTIFICATION}

While quantifying the predictive uncertainty for machine learning models, there are two broad classes of uncertainty that need to be considered: epistemic and aleatoric uncertainty. Epistemic uncertainty [52] is also referred to as reducible uncertainty or subjective uncertainty. Such uncertainty arises due to lack of knowledge regarding the dynamics of the system under consideration, or an inability to express it using models. Epistemic uncertainties are essential for safety critical applications, for instance to understand and identify examples that are significantly different from the training data [53]. Similarly, epistemic uncertainties are important while learning from small datasets where the training data is sparse, globally or locally. Epistemic uncertainty can be further divided into parameter uncertainty and structural uncertainty. Parameter uncertainty captures our ignorance about the exact combination of model coefficients that generated the training data. Such uncertainty can be minimized with larger corpora of training data. Structural uncertainty captures our inability to represent the underlying process via a limited model. In the context of deep learning, it quantifies the uncertainties in selection the nature of the NN, the optimal model architecture and other hyperparameters.

Aleatoric uncertainty [52] is often referred to as irreducible uncertainty or stochastic uncertainty. In real-life scientific applications, this arises due to noise in the training data, projection of data onto a lower space, absence of important features in the data, etc. While large datasets can ameliorate epistemic uncertainty, aleatoric uncertainties are immune to such remedies. Aleatoric uncertainties can be divided into homoscedastic uncertainty and heteroscedastic uncertainty. Homoscedastic uncertainties are 
independent of the input data but depend on the application. In contrast, heteroscedastic uncertainties strongly depend on the model input. These exhibit variability across examples with some inputs leading to significantly higher noise in the predictions for some samples than others. These two sources of uncertainty, epistemic and aleatoric, are significantly different in their nature and in the manner that they are dealt with in uncertainty quantification. For instance, Aleatoric uncertainties arise due to random variability and thus, introduce random variance in the model predictions. Epistemic uncertainties on the other hand arise due to incomplete representation of underlying physics and add a bias to the simulation results.

In physical sciences, the traditional divisions between different kinds of uncertainty and their definitions are different. Traditionally, uncertainty in physical sciences is demarcated into statistical and systematic uncertainties. Statistical uncertainties refer to errors that may be quantified by statistical analysis over a series of measurements. In contrast, systematic uncertainties, occur due to models and theory, and cannot be treated the same as statistical. As a mapping between the cognomen utilized in the different fields, epistemic uncertainties are always systematic uncertainties, and statistical uncertainties are always aleatoric.

\section{APPENDIX B: MATHEMATICAL DETAILS}

We emphasize that the Bayesian formalism is an approach (or algorithm), as opposed to a specific standalone model class. Bayesian approaches can be applied to most data-driven models, with rare exceptions such as treebased models. Herein, the weights and biases of the model would be represented as random variables with associated probability distributions, as opposed to deterministic scalars. Prior distributions are placed over these parameters. On observing data, these are converted to the posterior based on the Bayes' theorem.

Most classical models, for instance linear regression or logistic regression, have Bayesian counterparts, in this case Bayesian linear regression and Bayesian logistic regression. Similarly, the Bayesian counterpart for Kernel Regression (and classification) are Gaussian process-based regression (and classification). In the same vein, the Bayesian counterpart for deterministic NNs are referred to as BNNs.

\section{Mathematical formalism of Bayesian neural networks}

NNs are data-driven modeling algorithms for classification, regression, and ancillary applications. These include different neuron connectivity, architectures, etc., leading to different varieties of NNs such as fully connected NNs (FCNNs), convolutional NNs (CNNs), and recurrent NNs (RNNs), etc. Adhering to the fully connected NN as an archetype, the neurons of adjacent layers are densely connected, and outputs of a preceding layer are fed forward as inputs to the succeeding layer. In this vein, a fully connected $\mathrm{NN}$ defines a mapping from the space of the input layer, $z_{0} \in \mathbb{R}^{n_{0}}$, to the output layer, $z_{l} \in \mathbb{R}^{n_{l}}$. The layers between the input and output are referred to as "hidden layers" by convention. Mathematically, any two adjacent layers in such a network are connected via $z_{l}=g_{l}\left(W_{l}^{T} z_{l-1}+b_{l}\right)$, where $g_{l}$ denotes the activation function associated with the layer, for which a number of options can be chosen, for instance, sigmoid, ReLU, hyperbolic tangent, etc. $W_{l} \in \mathbb{R}^{n_{l-1} \times n_{l}}$ and $b_{l} \in \mathbb{R}^{n_{l}}$ are the weights and biases associated with the layer corresponding to the subscript $l$. As is denoted by the notation, these weights and biases are assumed to be deterministic vectors in the space of reals. The process of training the deterministic NN entails estimating the optimal values for these by minimizing an objective using maximum likelihood approaches.

In spite of this deterministic foundation, many researchers view NNs as inherently probabilistic models [54-56]. In this paradigm, given an input vector $x \in \mathbb{R}^{n_{0}}$, the NN assigns a conditional probability distribution, $P(y \mid x, W)$, over the set of possible outputs, $y$, parametrized by the weights and biases. For classification tasks, $P(y \mid x, W)$ corresponds to a categorical distribution. For regression tasks, $P(y \mid x, W)$ corresponds to a Gaussian distribution. Given training data in the from of labeled examples $D=\left(x_{i}, y_{i}\right)$, these weights and biases are learnt using maximum likelihood estimation, explicitly $W_{\mathrm{MLE}}=\arg \max _{W} \log P(D \mid W)$.

As was stated earlier, in contrast to classical deterministic NNs, in BNNs, the weights and biases are assumed to be random variables with corresponding probability distributions. Deterministic weights and biases lead to deterministic predictions in the output of the $\mathrm{NN}$. However, in the BNN the weights and biases are random variables with probability distributions, leading to an output prediction that has an associated probability distribution as well. Given training data, Bayesian inference over these BNNs estimates the posterior probability distributions for these weights and biases. While querying from such a trained BNN, the mean of predictions is given via expectations over the posterior distributions: $P\left(y^{*} \mid x^{*}\right)=E_{P(W \mid D)}\left(P\left(y^{*} \mid x^{*}, W\right)\right)$. Estimating the posterior distributions over the weights and biases for large networks was historically an intractable problem.

Prior researchers had proposed searching instead for a variational approximation to the Bayesian posterior probability distribution on the biases and weights $[54,55]$. Such variational approaches propose a family of parameterized distributions for the weights and biases, $q(W \mid \theta)$. Thence, the optimal value of the parameter, $\theta$ is approximated so as to minimize the Kullback-Leibler (KL) divergence between the parametrized distribution and the true Bayesian 
posterior probability distribution. Explicitly, this optimal parameter, $\theta^{*}$, is given by

$$
\begin{aligned}
\theta^{*} & =\underset{\theta}{\arg \min } K L(q(W \mid \theta) \| P(W \mid D)) \\
& =\underset{\theta}{\arg \min } \int q(W \mid \theta) \log \frac{q(W \mid \theta)}{P(W) P(D \mid W)} d W \\
& =\underset{\theta}{\arg \min } K L[q(W \mid \boldsymbol{\theta}) \| P(W)]-\mathbb{E}_{q(W \mid \boldsymbol{\theta})}[\log P(D \mid W)] .
\end{aligned}
$$

Using this as a surrogate objective function, the problem of stochastic inference is reduced to one concerning optimization. The resultant loss is often referred to as the variational free energy or the evidence lower bound and is given by

$$
F(D, \boldsymbol{\theta})=K L[q(W \mid \boldsymbol{\theta}) \| P(W)]-\mathbb{E}_{q(W \mid \boldsymbol{\theta})}[\log P(D \mid W)] .
$$

The first term of this objective is referred to as the complexity cost and depends on the priors used. The latter term is referred to as the likelihood cost and depends on the training data. In practice, approximations are utilized to simplify the expectation and the minimization is carried out via steepest descent. In this investigation, we carry out this optimization using Monte Carlo sampling in conjunction with local parameterization [37,57]. Herein, the objective function is estimated via Monte Carlo sampling via

$$
F(D, \boldsymbol{\theta}) \approx \sum_{i=1}^{n} \log q\left(W^{(i)} \mid \boldsymbol{\theta}\right)-\log P\left(W^{(i)}\right)-\log P\left(D \mid W^{(i)}\right),
$$

and $W^{(i)}$ represents the $i$ th Monte Carlo sample from the variational posterior. Following best practice, we use a standard normal as the prior on the weights of the network. The joint prior over the entire set of weights and biases of the network is given by the product of the individual independent normal distributions over individual parameters, $P(W)=\prod_{i} \mathcal{N}\left(W_{i} \mid 0,1\right)$. Our approach adheres to the Bayes By Backprop algorithm [37]. To optimize the variational parameters, we utilize the Adaptive Moment Estimation (Adam) algorithm, wherein the learning rate, the forgetting factors for gradients and second moments of gradients were set using cross validation.

\section{Mathematical formalism for Bootstrapped ensembles}

Deterministic NNs provide point estimates of the target but do not yield prediction intervals over the output. There are different techniques to extend these models in a non-Bayesian manner, including Naive ensembling [49], involving multiple random seeds and bagging ensembles [58,59] (or bootstrap aggregation). While using Naive ensembles, a number of different deterministic models are trained on different mini-batch orderings of the training data, with the weights and biases initialized to different values using different random seeds for each individual model. For problems with a nonconvex loss surface, such randomization can lead to the individual models in the ensemble pertaining to different minima on the loss surface. In the bootstrap aggregation approach, bootstrap datasets of $N$ samples each are engendered for training each individual model in the ensemble by sampling uniformly with replacement from the original training dataset of $N$ unique samples. Thence, individual NNs in the model ensemble are trained separately on each of these bootstrap datasets.

While making predictions in the regression context, the predictions of the individual trained models in the bootstrapped ensemble are averaged to get a mean prediction. Additionally, the variance in predictions from this bootstrapped ensemble of models provides a measure of the predictive uncertainty. In this investigation, we use bootstrapped ensembles with multiple random seeds. A key additional hyperparameter is the number of individual models in the ensemble. In each case, the number of individual models in the bootstrapped ensemble is increased till the metrics are constant.

[1] A. Edelen, S. Biedron, S. Milton, and J. Edelen, First steps toward incorporating image based diagnostics into particle accelerator control systems using convolutional neural networks, arXiv:1612.05662.

[2] E. Fol, Evaluation of machine learning methods for LHC optics measurements and corrections software, Ph.D. thesis, Hochschule, Eng. Econ., Karlsruhe, 2017.

[3] A. L. Edelen, S. Biedron, B. Chase, D. Edstrom, S. Milton, and P. Stabile, Neural networks for modeling and control of particle accelerators, IEEE Trans. Nucl. Sci. 63, 878 (2016).

[4] A. Edelen, J. Edelen, D. Bowring, B. Chase, D. Edstrom, J. Steimel, P. van der Slot, and S. Biedron, Recent applications of neural network-based approaches to the modeling and control of particle accelerators, in Proceedings of 9th International Particle Accelerator Conference (IPAC 2018) Vancouver, BC, Canada (JACoW, European Organization for Nuclear Research (CERN), Meyrin, 2018).

[5] A. Scheinker, C. Emma, A. Edelen, and S. Gessner, Advanced control methods for particle accelerators (ACM4PA) 2019, Technical Report No. LA-UR-1932526, Los Alamos National Laboratory (LANL), Los Alamos, NM (United States), 2019.

[6] X. Pang et al., Advances in proton linac online modeling, in Proceedings of IPAC'15 (JACoW, European Organization for Nuclear Research (CERN), Meyrin, 2015).

[7] C. Emma, A. Edelen, M. Hogan, B. O'Shea, G. White, and V. Yakimenko, Machine learning-based longitudinal phase 
space prediction of particle accelerators, Phys. Rev. Accel. Beams 21, 112802 (2018).

[8] A. L. Edelen, S. V. Milton, S. G. Biedron, J. P. Edelen, and P. J. van der Slot, Using a neural network control policy for rapid switching between beam parameters in an FEL, Technical Report No. LA-UR-17-28069, Los Alamos National Laboratory (LANL), Los Alamos, NM (United States), 2017.

[9] A. Scheinker and S. Gessner, Adaptive method for electron bunch profile prediction, Phys. Rev. ST Accel. Beams 18, 102801 (2015).

[10] J. Duris, D. Kennedy, A. Hanuka, J. Shtalenkova, A. Edelen, P. Baxevanis, A. Egger, T. Cope, M. McIntire, S. Ermon et al., Bayesian Optimization of a Free-Electron Laser, Phys. Rev. Lett. 124, 124801 (2020).

[11] A. Hanuka, J. Duris, J. Shtalenkova, D. Kennedy, A. Edelen, D. Ratner, and X. Huang, Online tuning and light source control using a physics-informed gaussian process Adi, arXiv:1911.01538.

[12] K. Hornik, Approximation capabilities of multilayer feedforward networks, Neural Netw. 4, 251 (1991).

[13] M. D. Zeiler and R. Fergus, Visualizing and understanding convolutional networks, in European Conference on Computer Vision (Springer, New York, 2014), pp. 818-833.

[14] X. Huang, M. Kwiatkowska, S. Wang, and M. Wu, Safety verification of deep neural networks, in International Conference on Computer Aided Verification (Springer, New York, 2017), pp. 3-29.

[15] R. Michelmore, M. Kwiatkowska, and Y. Gal, Evaluating uncertainty quantification in end-to-end autonomous driving control, arXiv:1811.06817.

[16] D. Amodei, C. Olah, J. Steinhardt, P. Christiano, J. Schulman, and D. Mané, Concrete problems in AI safety, arXiv:1606.06565.

[17] A. Nguyen, J. Yosinski, and J. Clune, Deep neural networks are easily fooled: High confidence predictions for unrecognizable images, in Proceedings of the IEEE conference on computer vision and pattern recognition (Institute of Electrical and Electronics Engineers (IEEE), Piscataway, 2015), pp. 427-436.

[18] D. Hendrycks and K. Gimpel, A baseline for detecting misclassified and out-of-distribution examples in neural networks, arXiv:1610.02136.

[19] K. Habib, Tesla crash preliminary evaluation report, Technical Report No. PE 16-007, National Highway Traffic Safety Administration, US Department of Transportation, 2017.

[20] L. Dodds, Chinese businesswoman accused of jaywalking after AI camera spots her face on an advert, Telegraph (Telegraph Media Group, London, 2018).

[21] N. T. S. B. (NTSB), Collision between a car operating with automated vehicle control systems and a tractor-semitrailer truck, Report No. NTSB/HAR-17-XX, Technical Report, National Transportation Safety Board, Washington, D.C., 2016.

[22] N. T. S. B. (NTSB), Collision between a sport utility vehicle operating with partial driving automation and a crash attenuator, Technical Report No. NTSB/HAR-20/01, National Transportation Safety Board, 2020.
[23] N. T. S. B. (NTSB), Collision between a car operating with automated vehicle control systems and a tractor-semitrailer truck, Technical Report No. NTSB/HAR-17/02, National Transportation Safety Board, Washington, D.C., 2017.

[24] U. Amaldi, The importance of particle accelerators, Europhysics News 31, 5 (2000).

[25] M. Fazio, G. Laramore, S. Pillai, A. Badruzzaman, H. Martz, J. Buchsbaum, D. Jaffray, M.-K. Boss, A. Schmidt, J. Calame et al., Basic research needs workshop on compact accelerators for security and medicine: Tools for the 21st century, 2019, Technical Report No. 1631121, USDOE Office of Science (SC), (United States), 2019.

[26] Z.-H. Zhou, Ensemble Methods: Foundations and Algorithms (CRC press, Boca Raton, 2012).

[27] N. R. Council, Scientific Assessment of High-Power FreeElectron Laser Technology (The National Academies Press, Washington, DC, 2009).

[28] J. F. Schultz, M. J. Lavan, E. W. Pogue, and T. W. Meyer, The high average power free electron laser program, Nucl. Instrum. Methods Phys. Res., Sect. A 318, 9 (1992).

[29] W. J. Ng, Design and analysis of megawatt class Free electron laser weapons, Technical Report No. 0704-0188, Naval Postgraduate School Monterey, United States, 2015.

[30] S. Benson, High power free-electron lasers, in Proceedings of the 1999 Particle Accelerator Conference (Cat. No. 99CH36366) (IEEE, Piscataway, 1999), Vol. 1, pp. 212216.

[31] I. Osband, C. Blundell, A. Pritzel, and B. Van Roy, Deep Exploration via Bootstrapped DQN, in Advances in neural information processing systems (MIT Press, Cambridge, Massachusetts, 2016), pp. 4026-4034.

[32] B. Lakshminarayanan, A. Pritzel, and C. Blundell, Simple and scalable predictive uncertainty estimation using deep ensembles, in Advances in Neural Information Processing Systems (MIT Press, Cambridge, Massachusetts, 2017), pp. 6402-6413.

[33] T. Pearce, A. Brintrup, M. Zaki, and A. Neely, High-quality prediction intervals for deep learning: A distribution-free, ensembled approach, in International Conference on Machine Learning (JMLR, Inc. and Microtome Publishing, United States, 2018), pp. 4075-4084.

[34] Y. Ovadia, E. Fertig, J. Ren, Z. Nado, D. Sculley, S. Nowozin, J. Dillon, B. Lakshminarayanan, and J. Snoek, Can you trust your model's uncertainty? evaluating predictive uncertainty under dataset shift, in Advances in Neural Information Processing Systems (MIT Press, Cambridge, Massachusetts, 2019), pp. 13991-14002.

[35] M. Hein, M. Andriushchenko, and J. Bitterwolf, Why relu networks yield high-confidence predictions far away from the training data and how to mitigate the problem, in Proceedings of the IEEE Conference on Computer Vision and Pattern Recognition (Institute of Electrical and Electronics Engineers (IEEE), Piscataway, 2019), pp. 41-50.

[36] Y. Gal, Uncertainty in Deep Learning (University of Cambridge, Cambridge, England, 2016), Vol. 1.

[37] C. Blundell, J. Cornebise, K. Kavukcuoglu, and D. Wierstra, Weight uncertainty in neural network, in International Conference on Machine Learning (Omnipress, Madison, 2015), pp. 1613-1622. 
[38] Y. Romano, E. Patterson, and E. J. Candès, Conformalized quantile regression, arXiv:1905.03222.

[39] R. Hettel, The completion of SPEAR 3, Technical Reports No. SLAC-PUB-11679, No. TRN: US0601022, Stanford Linear Accelerator Center (SLAC), Menlo Park, CA, 2005.

[40] A. Franchi, L. Farvacque, J. Chavanne, F. Ewald, B. Nash, K. Scheidt, and R. Tomás, Vertical emittance reduction and preservation in electron storage rings via resonance driving terms correction, Phys. Rev. ST Accel. Beams 14, 034002 (2011).

[41] P. Bellomo, R. Boyce, J. Corbett, P. Corredoura, E. Daly, D. Dell'Orco, T. Elioff, I. Evans, A. Fisher, and R. Hettel, SPEAR 3 design report, Technical Report No. SLAC-R609, No. TRN: US0302588, Stanford Linear Accelerator Center, Menlo Park, 1999.

[42] J. Safranek, Experimental determination of storage ring optics using orbit response measurements, Nucl. Instrum. Methods Phys. Res., Sect. A 388, 27 (1997).

[43] X. Huang, J. Corbett, J. Safranek, and J. Wu, An algorithm for online optimization of accelerators, Nucl. Instrum. Methods Phys. Res., Sect. A 726, 77 (2013).

[44] R. Ryne and S. Habib, Impact: An integrated-map \& particle accelerator tracking code for linac simulation, in American Physical Society, Particle Acceleration Meeting, 1997, abstract id. 9P.105 (1997).

[45] D. Sagan, Bmad: A relativistic charged particle simulation library, Computational accelerator physics, in Proceedings of 8th International Conference, ICAP 2004, St. Petersburg, Russia, 2004, Nucl. Instrum. Methods Phys. Res., Sect. A 558, 356 (2006).

[46] J. Quionero-Candela, M. Sugiyama, A. Schwaighofer, and N. D. Lawrence, Dataset Shift in Machine Learning (The MIT Press, Cambridge, Massachusetts, 2009).

[47] D. Hendrycks and T. Dietterich, Benchmarking neural network robustness to common corruptions and perturbations, in International Conference on Learning Representations (International Conference on Learning Representations, La Jolla, 2018).
[48] K. Floettmann, ASTRA: A space charge tracking algorithm, Technical Report No. DESY-3.2, Deutsches Elektronen-Synchrotron, Hamburg, Germany, 2017.

[49] I. Osband, Risk versus uncertainty in deep learning: Bayes, bootstrap and the dangers of dropout, in NIPS Workshop on Bayesian Deep Learning (MIT Press, Cambridge, Massachusetts, 2016), Vol. 192.

[50] J. Nixon, B. Lakshminarayanan, and D. Tran, Why are bootstrapped deep ensembles not better?, in "I Can't Believe It's Not Better!” NeurIPS 2020 workshop (2020).

[51] S. Lee, S. Purushwalkam, M. Cogswell, D. Crandall, and D. Batra, Why $M$ heads are better than one: Training a diverse ensemble of deep networks, arXiv:1511.06314.

[52] R. C. Smith, Uncertainty Quantification: Theory, Implementation, and Applications, Vol. 12 (Siam, Philadelphia, 2013).

[53] A. Kendall and Y. Gal, What uncertainties do we need in Bayesian deep learning for computer vision?, in Advances in neural information processing systems (MIT Press, Cambridge, Massachusetts, 2017), pp. 5574-5584.

[54] G. E. Hinton and D. Van Camp, Keeping the neural networks simple by minimizing the description length of the weights, in Proceedings of the 6th annual conference on Computational learning theory (1993), pp. 5-13.

[55] A. Graves, Practical variational inference for neural networks, in Advances in neural information processing systems (MIT Press, Cambridge, Massachusetts, 2011), pp. 2348-2356.

[56] I. Goodfellow, Y. Bengio, and A. Courville, Deep Learning (MIT press, Cambridge, Massachusetts, 2016).

[57] D. P. Kingma and M. Welling, Auto-encoding variational bayes, arXiv:1312.6114.

[58] L. Breiman, Bagging predictors, Mach. Learn. 24, 123 (1996).

[59] B. Efron, Bootstrap methods: Another look at the jackknife, in Breakthroughs in Statistics (Springer, New York, 1992), pp. 569-593. 